\title{
Moment-based boundary conditions for lattice Boltzmann magnetohydrodynamics
}

\author{
Paul J. Dellar
}

OCIAM, Mathematical Institute, 24-29 St Giles', Oxford, OX1 3LB, UK

\begin{abstract}
We present a moment-based approach for implementing boundary conditions in a lattice Boltzmann formulation of magnetohydrodynamics. Hydrodynamic quantities are represented using a discrete set of distribution functions that evolve according to a cut-down form of Boltzmann's equation from continuum kinetic theory. Electromagnetic quantities are represented using a set of vector-valued distribution functions. The nonlinear partial differential equations of magnetohydrodynamics are thus replaced by two constant-coefficient hyperbolic systems in which all nonlinearities are confined to algebraic source terms. Further discretising these systems in space and time leads to efficient and readily parallelisable algorithms. However, the widely used bounce-back boundary conditions place no-slip boundaries approximately half-way between grid points, with the precise position being a function of the viscosity and resistivity. Like most lattice Boltzmann boundary conditions, bounce-back is inspired by a discrete analogue of the diffuse and specular reflecting boundary conditions from continuum kinetic theory. Our alternative approach using moments imposes no-slip boundary conditions precisely at grid points, as demonstrated using simulations of Hartmann flow between two parallel planes.
\end{abstract}

Published 2013 as pages 83-90 of Numerical Mathematics and Advanced Applications 2011, Proceedings of ENUMATH 2011, the 9th European Conference on Numerical Mathematics and Advanced Applications, Leicester, September 2011, edited by A. Cangiani, R. L. Davidchack, E.Georgoulis, A. N. Gorban, J. Levesley \& M.V. Tretyakov.

DOI: $10.1007 / 978-3-642-33134-3+9$

(c) Springer-Verlag Berlin Heidelberg 2013

\section{Introduction}

The lattice Boltzmann approach to computational fluid dynamics is based on a disrete analogue of Boltzmann's equation from the kinetic theory of gases $[4,5,16,18]$. The particle velocity $\boldsymbol{\xi}$ is restricted to a discrete set $\boldsymbol{\xi}_{0}, \ldots, \boldsymbol{\xi}_{N}$. The corresponding distribution functions $f_{i}(\mathbf{x}, t)$ evolve according to the discrete Boltzmann equation

$$
\partial_{t} f_{i}+\boldsymbol{\xi}_{i} \cdot \nabla f_{i}=-\sum_{j=0}^{N} \Omega_{i j}\left(f_{j}-f_{j}^{(0)}\right)
$$

Hydrodynamic quantities such as the fluid density $\rho$, velocity $\mathbf{u}$, and momentum flux $\boldsymbol{\Pi}$ are given by moments of the $f_{i}$,

$$
\rho=\sum_{i=0}^{N} f_{i}, \quad \rho \mathbf{u}=\sum_{i=0}^{N} \boldsymbol{\xi}_{i} f_{i}, \quad \boldsymbol{\Pi}=\sum_{i=0}^{N} \boldsymbol{\xi}_{i} \boldsymbol{\xi}_{i} f_{i}, \quad \mathbf{Q}=\sum_{i=0}^{N} \boldsymbol{\xi}_{i} \boldsymbol{\xi}_{i} \boldsymbol{\xi}_{i} f_{i}
$$

These sums replace the integrals over $\boldsymbol{\xi}$ in classical kinetic theory [4]. The equilibrium distributions $f_{j}^{(0)}(\rho, \mathbf{u})$ and collision matrix $\Omega_{i j}$ are chosen so that slowly varying solutions of the moment hierarchy

$$
\partial_{t} \rho+\nabla \cdot(\rho \mathbf{u})=0, \quad \partial_{t}(\rho \mathbf{u})+\nabla \cdot \boldsymbol{\Pi}=0, \quad \partial_{t} \boldsymbol{\Pi}+\nabla \cdot \mathbf{Q}=-\frac{1}{\tau}\left(\boldsymbol{\Pi}-\boldsymbol{\Pi}^{(0)}\right)
$$

obtained from (1) satisfy the isothermal Navier-Stokes equations on timescales much longer than the timescale $\tau$ associated with collisions. We obtain the Euler equations with constant sound speed $c_{\mathrm{s}}$ by setting $\boldsymbol{\Pi}^{(0)}=c_{\mathrm{s}}^{2} \rho \mathbf{I}+\rho \mathbf{u u}$, where $\mathbf{I}$ is the identity tensor. The first correction to the momentum flux is given by

$$
\boldsymbol{\Pi}=\boldsymbol{\Pi}^{(0)}-\tau c_{\mathrm{s}}^{2} \rho\left((\nabla \mathbf{u})+(\nabla \mathbf{u})^{\top}\right)
$$

corresponding to a Newtonian viscous stress with dynamic viscosity $\mu=\tau c_{\mathrm{s}}^{2} \rho$.

The constant coefficient hyperbolic system (1) is readily discretised by integration along characteristics [12], or by splitting into separate advection and collision steps [8], to obtain the fully discrete system [7]

$$
\bar{f}_{i}\left(\mathbf{x}+\boldsymbol{\xi}_{i} \Delta t, t+\Delta t\right)=\bar{f}_{i}(\mathbf{x}, t)-\Delta t \sum_{j=0}^{N} \bar{\Omega}_{i j}\left(\bar{f}_{j}(\mathbf{x}, t)-f_{j}^{(0)}(\mathbf{x}, t)\right),
$$




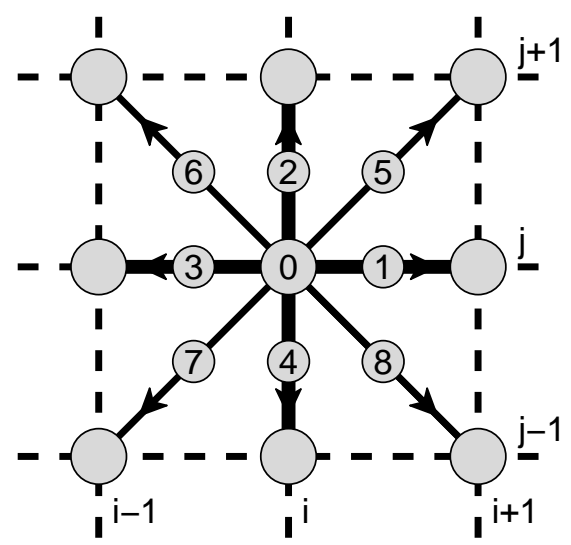

Fig. 1 Sketch of the discrete velocities $\boldsymbol{\xi}_{0}, \ldots, \boldsymbol{\xi}_{8}$ arranged on an integer lattice. Only the velocities $\boldsymbol{\xi}_{0}, \ldots, \boldsymbol{\xi}_{4}$ shown with thicker arrows are used for the magnetic distribution functions.

under the change of variables $\bar{f}_{i}=f_{i}+\frac{1}{2} \Delta t \sum_{j=0}^{N} \Omega_{i j}\left(f_{j}-f_{j}^{(0)}\right)$. The discrete collision matrix is $\bar{\Omega}=\left(\mathbf{I}+\frac{1}{2} \Delta t \boldsymbol{\Omega}\right)^{-1} \boldsymbol{\Omega}$. These formulae reduce to the standard redefinition of the collision time from $\tau$ to $\tau+\Delta t / 2$ for the single-relaxation-time collision operator $\Omega_{i j}=\tau^{-1} \delta_{i j}$.

\section{The magnetic field}

Magnetohydrodynamics describes the interaction between electrically conducting fluids and magnetic fields [3, 14]. The Lorentz force exerted by a magnetic field $\mathbf{B}$ may be expressed as the divergence of the Maxwell stress $\frac{1}{2}|\mathbf{B}|^{2} \mathbf{I}-\mathbf{B B}$. These two terms give an isotropic magnetic pressure and a tension directed along field lines. The Lorentz force is thus readily incorporated into lattice Boltzmann hydrodynamics by choosing the equilibrium momentum flux to be [6]

$$
\boldsymbol{\Pi}^{(0)}=c_{\mathrm{s}}^{2} \rho \mathbf{I}+\rho \mathbf{u u}+\frac{1}{2}|\mathbf{B}|^{2} \mathbf{I}-\mathbf{B B} .
$$

Suitable two-dimensional equilibria (in units where $c_{\mathrm{s}}=1 / 3$ ) are given by

$$
f_{i}^{(0)}=w_{i}\left[\rho\left(2-\frac{3}{2}\left|\boldsymbol{\xi}_{i}\right|^{2}\right)+3 \rho \mathbf{u} \cdot \boldsymbol{\xi}_{i}+\frac{9}{2} \boldsymbol{\Pi}^{(0)}: \boldsymbol{\xi}_{i} \boldsymbol{\xi}_{i}-\frac{3}{2} \operatorname{Tr} \boldsymbol{\Pi}^{(0)}\right] .
$$

The discrete velocities $\boldsymbol{\xi}_{0}, \ldots, \boldsymbol{\xi}_{8}$ form an integer square lattice in these units, see figure 1. The corresponding weights are $w_{0}=4 / 9, w_{1,2,3,4}=1 / 9$, and $w_{5,6,7,8}=1 / 36$. The expressions (7) reduce to the standard D2Q9 equilibria [16] when $\mathbf{B}=0$.

The magnetic field evolves through Faraday's law $\partial_{t} \mathbf{B}+\nabla \times \mathbf{E}=0$, where the electric field $\mathbf{E}$ is given by Ohm's law $\mathbf{E}+\mathbf{u} \times \mathbf{B}=\eta \nabla \times \mathbf{B}$ in resistive magnetohydrodynamics. Faraday's law cannot be derived from a kinetic equation of the form (1), because the vector $\rho \mathbf{u}$ evolves through the divergence of the symmetric tensor $\boldsymbol{\Pi}$ in (3). By contrast, $\partial_{t} \mathbf{B}=-\nabla \cdot \boldsymbol{\Lambda}$ evolves through the divergence of an antisymmetric tensor whose components $\Lambda_{\alpha \beta}=-\epsilon_{\alpha \beta \gamma} E_{\gamma}$ are formed by contracting the electric field with the alternating tensor.

Instead, we represent the magnetic field as $\mathbf{B}=\sum_{i} \mathbf{g}_{i}$ using a set of vector-valued distribution functions $\mathbf{g}_{i}$ that evolve according to the vector Boltzmann equation [6]

$$
\partial_{t} \mathbf{g}_{i}+\boldsymbol{\xi}_{i} \cdot \nabla \mathbf{g}_{i}=-\frac{1}{\tau_{m}}\left(\mathbf{g}_{i}-\mathbf{g}_{i}^{(0)}\right)
$$

with the equilibrium distributions

$$
\mathbf{g}_{i}^{(0)}=W_{i}\left(\mathbf{B}-3 \boldsymbol{\xi}_{i} \times(\mathbf{u} \times \mathbf{B})\right) .
$$

The magnetic weights are $W_{0}=1 / 3$ and $W_{1,2,3,4}=1 / 6$. The four diagonal velocities $\boldsymbol{\xi}_{5}, \ldots, \boldsymbol{\xi}_{8}$ are not needed for the magnetic distribution functions. [6]

Slowly varying solutions of (8) obey the correct evolution equation for a magnetic field under resistive magnetohydrodynamics

$$
\partial_{t} \mathbf{B}=\nabla \times(\mathbf{u} \times \mathbf{B})+\nabla \cdot(\eta \nabla \mathbf{B}),
$$

with resistivity given by $\eta=\tau_{m} / 3$ in the so-called lattice units with $\left|\boldsymbol{\xi}_{1,2,3,4}\right|=1$. Discretising (8) leads to a numerical scheme analogous to (5) that is coupled to the hydrodynamic lattice Boltzmann equation through the macroscopic velocity and magnetic field at grid points. The resulting numerical scheme preserves $\nabla \cdot \mathbf{B}=0$ to round-off error. It has been used in large-scale (up to $1800^{3}$ grid points) simulations of three-dimensional MHD turbulence [19, 17], and to simulate liquid metal flows in cooling systems for nuclear reactors[15]. 

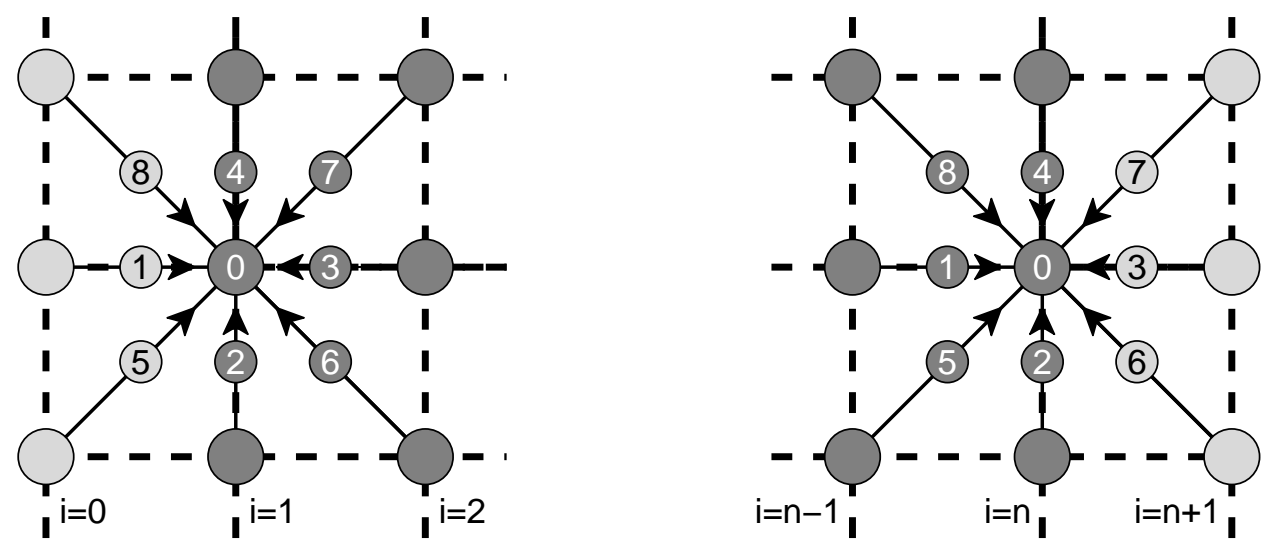

Fig. 2 The boundary conditions must supply values for the incoming distributions $f_{1}, f_{5}, f_{8}$ on the left boundary, and for $f_{3}, f_{6}, f_{7}$ on the right boundary.

\section{Boundary conditions for Hartmann flow}

We present simulations of the MHD analogue of Poiseuille flow, known as Hartmann flow, in which a uniform pressure gradient drives a unidirectional flow along a channel spanned by an imposed uniform magnetic field. The flow stretches the imposed field to create an additional magnetic field component along the channel, and hence a Lorentz force that resists the flow. We choose axes with $y$ directed along the channel, and $x$ directed across the channel with the walls at $x= \pm L$. Imposing no-flux and no-slip boundary conditions corresponds to setting $\mathbf{u}=0$ on the walls. Maxwell's equations imply continuity of the normal and tangential components of $\mathbf{B}$ at the walls, so $\mathbf{B}=\left(B_{0}, 0,0\right)$ takes its external applied value [14].

Following the approach of Bennett $[1,2]$ we formulate boundary conditions in terms of moments of the $f_{i}$. At the left-hand boundary we must supply values for the three distributions $f_{1}, f_{5}, f_{8}$ that propagate inwards from outside the domain, as sketched in figure 2. This may be done by specifying values for the three moments $\rho u_{x}, \rho u_{y}$, and $\Pi_{y y}$,

$$
\begin{aligned}
& 0=\rho u_{x}=f_{1}+f_{5}+f_{8}-f_{3}-f_{6}-f_{7}, \\
& 0=\rho u_{y}=f_{5}-f_{8}+f_{2}-f_{4}+f_{6}-f_{7}, \\
& \Pi_{y y}^{(0)}=\Pi_{y y}=f_{5}+f_{8}+f_{2}+f_{4}+f_{6}+f_{7} .
\end{aligned}
$$

These three moments are chosen because they contain three linearly independent combinations of the unknowns $f_{1}, f_{5}, f_{8}$. The first two conditions (11a) and (11b) impose no-flux and no-slip boundary conditions, and the third boundary condition (11c) on the tangential stress has a more natural physical interpretation than the alternatives involving the higher moments [1,2]. Solving this system of three linear equations determines the incoming distributions,

$$
\begin{aligned}
& f_{1}=f_{2}+f_{3}+f_{4}+2 f_{6}+2 f_{7}-\Pi_{y y}^{(0)}, \\
& f_{5}=-f_{2}-f_{6}+\frac{1}{2} \Pi_{y y}^{(0)}, \\
& f_{8}=-f_{4}-f_{7}+\frac{1}{2} \Pi_{y y}^{(0)} .
\end{aligned}
$$

For this simple flow it is sufficient to take $\Pi_{y y}^{(0)}=c_{\mathrm{s}}^{2} \rho=c_{\mathrm{s}}^{2}$ on the boundary, since the tangential velocity and magnetic field both vanish. The fluid density $\rho$ is uniform, and may be set equal to unity in the initial conditions. More generally, one would solve for $\rho$ as part of the linear system by setting $\Pi_{y y}^{(0)}=c_{\mathrm{s}}^{2}\left(f_{0}+\cdots+f_{8}\right)$ in $(11 \mathrm{c})$

A similar approach determines the incoming magnetic distributions $g_{1 x}$ and $g_{1 y}$ from the boundary conditions $B_{x}=B_{0}$ and $B_{y}=0$,

$$
\begin{aligned}
& B_{x}=B_{0} \quad \Longrightarrow g_{x 1}=B_{0}-\left(g_{x 0}+g_{x 2}+g_{x 3}+g_{x 4}\right) \\
& B_{y}=0 \quad \Longrightarrow g_{y 1}=\quad-\left(g_{y 0}+g_{y 2}+g_{y 3}+g_{y 4}\right)
\end{aligned}
$$

The same approach enables $f_{3}, f_{6}, f_{7}$ and $g_{x 3}, g_{y 3}$ to be determined at the right-hand wall, and we impose periodic boundary conditions in the $y$ direction.

\section{Numerical experiments}

Figure 3 shows the results of a lattice Boltzmann computation using these boundary conditions. The flow was driven by including an additional linear stress $x F \hat{\mathbf{x}} \hat{\mathbf{y}}$ into $\boldsymbol{\Pi}^{(0)}$, equivalent to a uniform body force $F \hat{\mathbf{y}}$, as in previous computations [6, 9]. The 

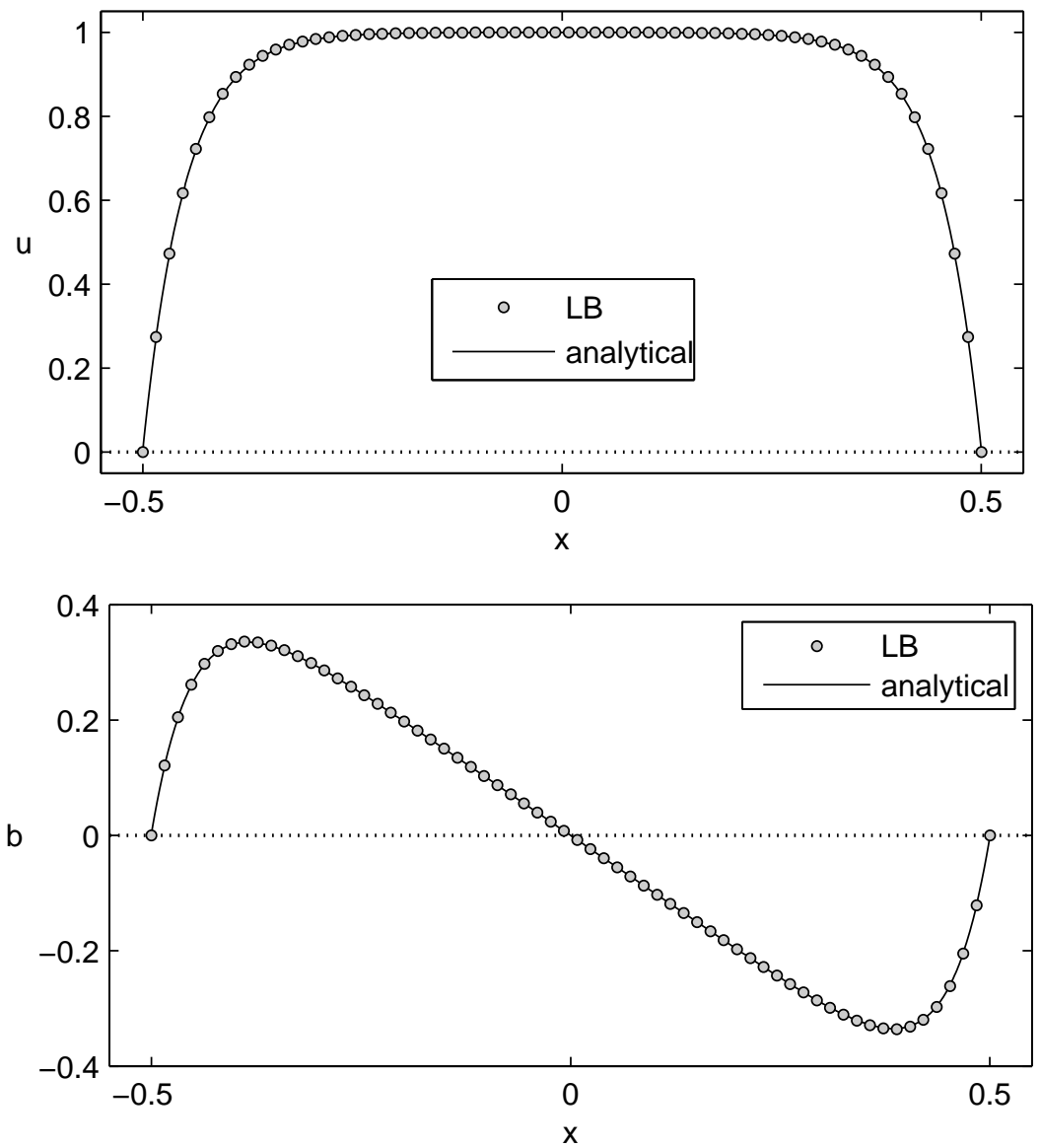

Fig. 3 Streamwise velocity and magnetic field, lattice Boltzmann (LB) computations versus the analytical solution for Hartmann number $H=10$, $B_{0}=1$, and 64 points. The velocity and magnetic field both vanish up to round-off error at the endpoints.

channel was taken to be the domain $|x| \leq L=0.5$ in suitable dimensionless units, with $B_{0}=1$ and $F=1$. The resistivity was $\eta=0.1$ and the kinematic viscosity was $\nu=\mu / \rho=0.025$. The lattice Boltzmann simulation shown was performed with 64 points and Mach number $\mathrm{Ma}=\sqrt{3} / 50$. The Mach number controls the ratio between the macroscopic fluid speed and the particle speeds, since $c_{\mathrm{s}}=\left|\boldsymbol{\xi}_{1}\right| / \sqrt{3}$.

Incompressible Hartmann flow in fluid of unit density has the exact solution [14]

$$
b(x)=\frac{F L}{B_{0}}\left[\frac{\sinh (H x / L)}{\sinh (H)}-\frac{x}{L}\right], u(x)=\frac{F L}{B_{0}} \sqrt{\frac{\eta}{\nu}} \operatorname{coth}(H)\left[1-\frac{\cosh (H x / L)}{\cosh (H)}\right]
$$

for the streamwise ( $y$-component) velocity and magnetic field, while the spanwise magnetic field remains uniform. The Hartmann number $H=B_{0} L /(\eta \nu)^{1 / 2}$ measures the ratio of Lorentz to viscous forces, with $H=10$ for the parameters given. When $H \gg 1$ the streamwise velocity is nearly uniform, and the magnetic field nearly linear, outside $O(L / H)$-wide boundary layers at the walls, as shown in figure 3 .

The streamwise velocity and magnetic field both vanish precisely at the end-points using moment-based boundary conditions, unlike previous computations using bounce-back boundary conditions [6, 9]. Figure 4 shows the discrete $\ell_{2}$ norms of the differences between the lattice Boltzmann (LB) and analytical solutions,

$$
\Delta u=\left(\frac{1}{n} \sum_{i=1}^{n}\left|u_{L B}\left(x_{i}\right)-u\left(x_{i}\right)\right|^{2}\right)^{1 / 2}, \quad \Delta b=\left(\frac{1}{n} \sum_{i=1}^{n}\left|b_{L B}\left(x_{i}\right)-b\left(x_{i}\right)\right|^{2}\right)^{1 / 2},
$$

for different numbers of grid points $n$. To achieve the expected second-order convergence rate with the simple linear forcing term in the equilibrium stress it was necessary to decrease the Mach number Ma $=\sqrt{3} \times 2.56 / n$ with increasing $n$.

\section{Conclusion}

Restricting the particle velocity $\boldsymbol{\xi}$ in the Boltzmann equation to a discrete set $\boldsymbol{\xi}_{0}, \ldots, \boldsymbol{\xi}_{N}$ leads to a tractable system of partial differential equations for distribution functions $f_{i}(\mathbf{x}, t)$. Discretising these equations in $\mathbf{x}$ and $t$ leads to an effective tool for 


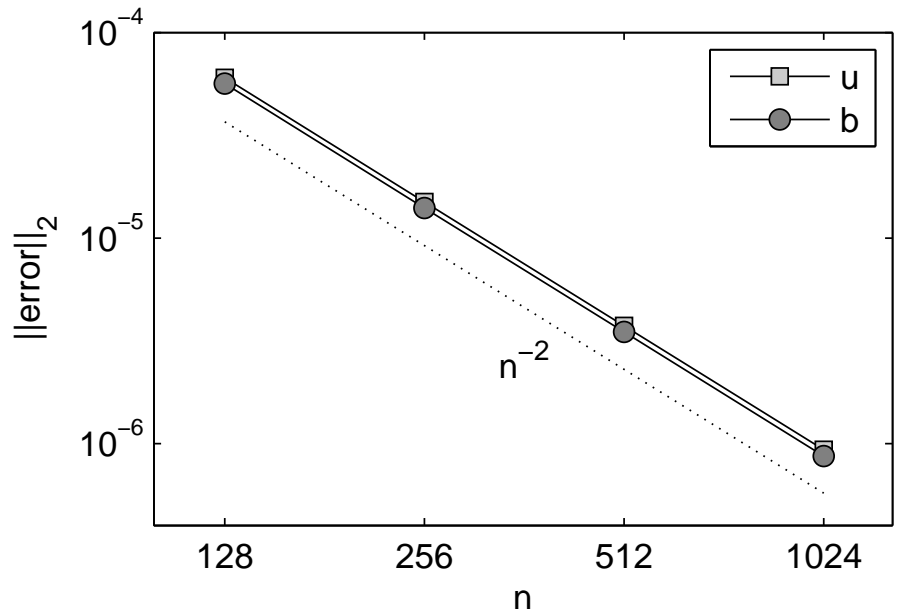

Fig. 4 Second-order convergence of the computed velocity and magnetic field towards the analytical solution (14) with increasing number of grid points

computational fluid dynamics. However, the widely used bounce-back boundary conditions, inspired by the diffuse and specular reflection of continuum kinetic theory, only approximate no-slip boundary conditions. The tangential velocity vanishes at a point approximately half-way between grid points, but the precise location depends on the collision rate $\tau$ unless one adopts a two-relaxation-time (TRT) collision operator with a specific ratio of relaxation times for odd and even moments [11, 13, 10]. The alternative approach of Bennett $[1,2]$ formulates boundary conditions for moments with direct physical interpretations, the velocity components and the tangential momentum flux. Solving the resulting linear system for the incoming distribution functions imposes no-slip and no-flux boundary conditions precisely at grid points. This approach extends easily to impose boundary conditions on a magnetic field, as shown for simulations of Hartmann flow between planar boundaries.

\section{Acknowledgement}

The author's research was supported by an Advanced Research Fellowship from the Engineering and Physical Sciences Research Council [grant number EP/E054625/1].

\section{References}

1. Bennett, S.: A lattice Boltzmann model for diffusion of binary gas mixtures. Ph.D. thesis, University of Cambridge (2010). Available from http://www.dspace.cam.ac.uk/handle/1810/226851.

2. Bennett, S., Asinari, P., Dellar, P.J.: A lattice Boltzmann model for diffusion of binary gas mixtures that includes diffusion slip. Int. J. Numer. Meth. Fluids 69, 171-189 (2012).

3. Biskamp, D.: Nonlinear Magnetohydrodynamics. Cambridge University Press, Cambridge (1993).

4. Chapman, S., Cowling, T.G.: The Mathematical Theory of Non-Uniform Gases, 3rd edn. Cambridge University Press, Cambridge (1970).

5. Chen, S., Doolen, G.D.: Lattice Boltzmann method for fluid flows. Annu. Rev. Fluid Mech. 30, 329-364 (1998).

6. Dellar, P.J.: Lattice kinetic schemes for magnetohydrodynamics. J. Comput. Phys. 179, 95-126 (2002).

7. Dellar, P.J.: Incompressible limits of lattice Boltzmann equations using multiple relaxation times. J. Comput. Phys. 190, 351-370 (2003).

8. Dellar, P.J.: An interpretation and derivation of the lattice Boltzmann method using Strang splitting. Comput. Math. Applic. 65, 129-141 (2013).

9. Dellar, P.J.: Lattice Boltzmann formulation for Braginskii magnetohydrodynamics. Computers \& Fluids 46, 201-205 (2011).

10. d'Humières, D., Ginzburg, I.: Viscosity independent numerical errors for Lattice Boltzmann models: From recurrence equations to "magic" collision numbers. Comput. Math. Applic. 58, 823-840 (2009).

11. Ginzbourg, I., Adler, M.P.: Boundary flow condition analysis for the three-dimensional lattice Boltzmann model. J. Phys. II France 4, 191-214 (1994).

12. He, X., Chen, S., Doolen, G.D.: A novel thermal model of the lattice Boltzmann method in incompressible limit. J. Comput. Phys. 146, 282-300 (1998).

13. He, X.Y., Zou, Q.S., Luo, L.S., Dembo, M.: Analytic solutions of simple flows and analysis of nonslip boundary conditions for the lattice Boltzmann BGK model. J. Statist. Phys. 87, 115-136 (1997).

14. Landau, L.D., Lifshitz, E.M.: Electrodynamics of Continuous Media. Pergamon, Oxford (1960). 2nd edition 1984.

15. Pattison, M., Premnath, K., Morley, N., Abdou, M.: Progress in lattice Boltzmann methods for magnetohydrodynamic flows relevant to fusion applications. Fusion Eng. Design 83, 557-572 (2008).

16. Qian, Y.H., d'Humières, D., Lallemand, P.: Lattice BGK models for the Navier-Stokes equation. Europhys. Lett. 17, 479-484 (1992).

17. Riley, B., Richard, J., Girimaji, S.S.: Assessment of magnetohydrodynamic lattice Boltzmann schemes in turbulence and rectangular jets. Int. J. Mod. Phys. C 19, 1211-1220 (2008).

18. Succi, S.: The Lattice Boltzmann Equation: For Fluid Dynamics and Beyond. Oxford University Press, Oxford (2001).

19. Vahala, G., Keating, B., Soe, M., Yepez, J., Vahala, L., Carter, J., Ziegeler, S.: MHD turbulence studies using lattice Boltzmann algorithms. Commun. Comput. Phys. 4, 624-646 (2008). 